\title{
JÓVENES Y USO PROBLEMÁTICO DE LAS TECNOLOGÍAS DURANTE LA PANDEMIA: UNA PREOCUPACIÓN FAMILIAR
}

\author{
YOUNG PEOPLE AND PROBLEMATIC USE OF TECHNOLOGIES DURING \\ THE PANDEMIC: A FAMILY CONCERN
}

\author{
JOVENS E USO PROBLEMÁTICO DE TECNOLOGIAS DURANTE A \\ PANDEMIA: UMA PREOCUPAÇAO FAMILIAR
}

\author{
Andrea Cívico Ariza \\ Universidad Internacional de Valencia, España \\ https://orcid.org/0000-0003-3094-5841 \\ andrea.civico@campusviu.es \\ Nuria Cuevas Monzonís \\ Universidad Internacional de Valencia, España \\ https://orcid.org/0000-0001-9366-3038 \\ nuria.cuevas@campusviu.es \\ Ernesto Colomo Magaña \\ Universidad de Málaga, España \\ https://orcid.org/0000-0002-3527-7937 \\ ecolomo@uma.es \\ Vicente Gabarda Méndez \\ Universitat de València, España \\ https://orcid.org/0000-0001-6159-5173 \\ vicente.gabarda@uv.es
}

Recibido: 22/02/2021 Revisado: 11/03/2021 Aceptado: 15/03/2021 Publicado: 22/03/2021

Resumen: La situación de confinamiento, derivada de la pandemia mundial por la COVID-19, ha supuesto un cambio drástico en nuestras conductas en las diferentes esferas de nuestra vida. Así, el desempeño de las actividades laborales, el afrontamiento de las responsabilidades académicas y la ocupación del tiempo de ocio se han visto condicionadas por una limitación de la movilidad y una mayor permanencia en el hogar. El confinamiento se ha vinculado a un mayor consumo de tecnología en sus diferentes formatos, generando una preocupación en el entorno familiar por un uso excesivo de ellas, así como de las posibles consecuencias que podrían derivarse. Bajo esta perspectiva, el presente estudio pretende conocer la percepción de 147 estudiantes de la Universidad de Málaga sobre la preocupación de sus familias respecto al uso de la tecnología (internet, móvil, videojuegos, mensajería instantánea y redes sociales) antes y durante el confinamiento. Los datos, recogidos mediante un instrumento validado, arrojan que hay una mayor preocupación familiar por la utilización de las diferentes tecnologías durante el confinamiento y que hay diferencias en función del género, siendo mayor la preocupación por el consumo del móvil y la mensajería instantánea en las mujeres y por los videojuegos en los hombres.

Palabras claves: Educación; Tecnologías; Familia; Estudiantes universitarios.

Abstract: The situation of confinement, derived from the global pandemic caused by COVID19 , has led to a drastic change in our behaviors in the different spheres of our life. Thus, the 
performance of work activities, the realization of academic responsibilities and the occupation of leisure time have been conditioned by limited mobility and a greater stay in the home. Confinement has been linked to a greater consumption of technology in its different formats, generating concern in the family environment for an excessive use of them, as well as the possible consequences that could result. Under this perspective, this study aims to know the perception of 147 students from the University of Malaga about the concern of their families regarding the use of technology (internet, cell phones, video games, instant messaging and social networks) before and during confinement. The data, collected through a validated instrument, show that there is a greater family concern about the use of technologies during the confinement and that there are gender-based differences, with greater concern about the use of cell phones and instant messaging in women and video games in men.

Keywords: Education; Technologies; Family; University students.

Resumo: A situação de confinamento, derivada da pandemia global causada pela COVID-19, significou uma mudança drástica no nosso comportamento nas diferentes esferas das nossas vidas. Assim, o desempenho de actividades de trabalho, a assunção de responsabilidades académicas e a ocupação de tempos livres foram condicionados por uma mobilidade limitada e uma maior permanência em casa. O confinamento tem estado ligado a um maior consumo de tecnologia nos seus diferentes formatos, gerando uma preocupação no ambiente familiar pela sua utilização excessiva, bem como as possíveis consequências que poderiam ser derivadas. Sob esta perspectiva, o presente estudo visa conhecer a percepção de 147 estudantes da Universidade de Málaga sobre a preocupação das suas famílias relativamente ao uso da tecnologia (internet, telemóvel, jogos de vídeo, mensagens instantâneas e redes sociais) antes e durante o confinamento. Os dados, recolhidos através de um instrumento validado, mostram que existe uma maior preocupação familiar sobre a utilização de diferentes tecnologias durante o confinamento e que existem diferenças de acordo com o sexo, com maior preocupação sobre a utilização de telemóveis e mensagens instantâneas nas mulheres e jogos de vídeo nos homens

Palavras-chave: Educação; Tecnologias; Família; Estudantes universitários.

Cómo citar este artículo: Cívico, A., Cuevas, N., Colomo, E. y Gabarda, V. (2021). Jóvenes y uso problemático de las tecnologías durante la pandemia: una preocupación familiar. Hachetetepé. Revista científica en Educación y Comunicación, (22), 1-12. https://doi.org/10.25267/Hachetetepe.2021.i22.1204

\section{INTRODUCCIÓN}

La situación mundial de pandemia, derivada de la COVID-19, está modificando algunos de nuestros patrones de conducta de manera drástica. Los procesos de socialización, el desarrollo de las tareas profesionales o el modo en que se implementan los procesos formativos de cualquier etapa han tenido que adaptarse a una nueva realidad donde la presencia física en los diferentes escenarios supone un riesgo para la salud.

De este modo, los recursos y herramientas que ayudan a suplir (aunque sea en parte) esta presencialidad, han ido ganando peso hasta convertirse prácticamente en un requisito imprescindible para continuar con nuestra vida cotidiana en sus vertiente social, profesional y académica. Estas herramientas se materializan de manera específica en las Tecnologías de la Información y la Comunicación (en adelante, TIC) que, teniendo formatos y usos diferentes en los diversos contextos, han facilitado la transición de lo presencial a lo digital, permitiendo retomar las diferentes actividades. 
Esta transición conlleva, de un modo implícito y necesario, una mayor utilización de las TIC, al convertirse en un elemento sine qua non para el desarrollo de nuestras obligaciones y necesidades en situaciones de confinamiento.

\subsection{Patrones de uso de la tecnología en tiempos de pandemia: panorama general}

En las últimas décadas, la tecnología se ha integrado en nuestras vidas de manera gradual y vinculándose, cada vez más, a nuestra relación con el entorno en diferentes esferas. De este modo, la inicial inclusión en nuestros contextos laborales, ligada a procesos de automatización de algunos procesos o como recurso para potenciarlos, ha dado paso, en la actualidad, al teletrabajo como realidad laboral y a una tecnologización de gran parte de las tareas que se vinculan al desempeño de los puestos de trabajo, independientemente del sector (Instituto Nacional de Estadística, 2020).

El ámbito educativo, obviamente, no ha sido ajeno a este proceso tecnológico. Desde mediados de los años 80 los centros educativos se han ido dotando de equipamiento que ha seguido un proceso de integración didáctica y curricular creciente, hasta dar lugar a modalidades de educación híbrida o en línea donde la tecnología ya no puede ser considerada un soporte, sino en el elemento central de la propia acción formativa. Estas modalidades, que hasta hace un año suponían experiencias casi aisladas y ligadas a modelos de negocio específicos, se han convertido, a raíz de la situación de pandemia y su confinamiento derivado, en una realidad constatable en las aulas de cualquier etapa educativa de nuestro país. De este modo, y de manera abrupta, los centros educativos y sus docentes han tenido que adaptar sus programaciones y sus prácticas a una modalidad en línea (Hodges et al., 2020) que posibilitara la continuidad de los procesos de enseñanza y aprendizaje del alumnado. Aunque este cambio de modalidad ha supuesto diversos modos y niveles de hibridación en las etapas preuniversitarias, en el caso de la educación superior ha servido para poner de manifiesto la necesidad de transformación digital, así como de una reflexión profunda sobre sus metodologías (García-Peñalvo y Corell, 2020).

Otra esfera que ha cambiado de manera radical es la del ocio. Las restricciones de movilidad y de vida social han potenciado nuevos patrones de socialización y de utilización del tiempo libre. Por un lado, ha habido un aumento muy considerable de la utilización de las redes sociales o los servicios de mensajería instantánea (Dong et al., 2020), convirtiéndolas en uno de los principales medios para la comunicación y la construcción de relaciones interpersonales. Este crecimiento ha supuesto una modificación de nuestros patrones de ocio, haciendo que dediquemos mucho más tiempo a la utilización del teléfono móvil con fines de entretenimiento tanto en redes sociales como en otras cuestiones como los juegos en línea y videojuegos (Lemenager et al., 2021). Es reseñable, además, que, pese a lo generalizado de la situación, el consumo parece estar determinado por el género, siendo las mujeres quienes hacen una mayor utilización de los dispositivos móviles (Chóliz et al., 2016; Fernández et al., 2020), mientras que los hombres hacen un mayor uso de los videojuegos (Desai et al., 2010).

\subsection{El papel de las familias en el uso de las tecnologías en el hogar}

Tal y como hemos podido analizar hasta ahora, el confinamiento ha supuesto un aumento de la tecnología por parte de toda la población, un consumo que se ha realizado de manera casi exclusiva en el ámbito privado del hogar. Aunque bien es cierto que la ubicuidad que proporcionan los dispositivos móviles facilitan su utilización en cualquier contexto y situación, el ámbito familiar supone uno de las esferas donde se produce gran 
parte de su utilización, pudiendo tener repercusiones en la relación y convivencia con los familiares con quien se reside (Muros et al., 2013).

Previamente al confinamiento, esta cuestión se vinculaba especialmente al uso de internet y los videojuegos, pero actualmente es extensible al resto de tecnologías que se utilizan en el hogar (dispositivos móviles, mensajería instantánea y redes sociales). Su consumo se vincula con el tiempo de ocio de carácter individual, siendo una actividad no compartida con el núcleo familiar ni supervisada por él, una cuestión que genera preocupación y desasosiego especialmente a los progenitores (Bringué et al., 2013). Es una cuestión lógica si tenemos en cuenta que la institución familiar es, por definición, un espacio de socialización y construcción de creencias, valores y normas de conducta (Cánovas y Sahuquillo, 2014), una labor que también han asumido en los últimos tiempos los medios de comunicación (Leoz, 2015).

Esta preocupación, más allá de su vinculación con la disrupción de unos patrones de convivencia establecidos, pueden vincularse con posibles problemas de salud, de conducta y de socialización (Ricoy y Ameneiros, 2016) y, en última instancia, con el fenómeno creciente de adicción a las tecnologías (Gonzálvez et al., 2017).

Igualmente, los patrones de consumo y niveles de competencia digital diferenciales entre las generaciones convivientes, puede suponer otro punto de fricción en la convivencia, siendo necesario implementar propuestas de intervención que establezcan pautas de alfabetización mediática en el contexto familiar (GonzálezFernández et al., 2019), que les permita conocer algunas premisas básicas para el uso seguro de la tecnología (Labrador et al., 2015), así como para integrarlas en la dinámica del hogar de un modo natural (López y González, 2017).

Partiendo de todo lo expuesto, el propósito de este estudio es analizar la percepción de las familias respecto al uso de las TIC por parte de los estudiantes universitarios antes y durante el confinamiento. De forma específica, se comprobará si existen diferencias significativas en la percepción de consumo excesivo por parte de las familias en función del sexo de los estudiantes en ambos momentos contemplados.

\section{METODOLOGÍA}

A fin de dar respuesta a este propósito, se detalla a continuación el planteamiento metodológico del estudio, explicitando el enfoque, la composición de la muestra, el instrumento utilizado para la recogida de información, el procedimiento seguido y la descripción del análisis de datos realizado.

\subsection{Enfoque metodológico}

Se realizó un estudio cuantitativo cuasiexperimental (grupos no aleatorios) con un diseño longitudinal de medidas pre-test y post-test. Se recogió información sobre el uso y consumo problemático de las TIC antes y durante el confinamiento provocado por la COVID-19, realizando análisis descriptivos e inferenciales considerando la variable sexo de los participantes.

\subsection{Muestra}

Partiendo de un muestreo intencional (no probabilístico) durante el curso 2019/2020, los participantes del estudio fueron 147 alumnos/as de la Universidad de Málaga de la Facultad de Ciencias de la Educación. Con una edad media de 20.52 \pm 1.77 años, 107 fueron mujeres $(72.79 \%)$ y 40 fueron hombres $(27.71 \%)$. Respecto a las 
titulaciones, 90 pertenecían al Grado en Educación Primaria (61.22 \%), 24 al Grado en Educación Infantil (16.33 \%), 20 al Grado en Educación Social (13.61 \%) y 13 al Grado en Pedagogía $(8.84 \%)$.

\subsection{Instrumento}

Se utilizó una sección del instrumento validado MULTICAGE-TIC (PedreroPérez et al., 2018). Este cuestionario posee propiedades psicométricas adecuadas, alcanzando resultados aceptables y excelentes respecto a la fiabilidad (internet, $\alpha=0.80$; móvil, $\alpha=0.72$; videojuegos, $\alpha=0.90$; mensajería instantánea, $\alpha=0.89$; redes sociales, $\alpha=0.93$ ), y una validez tanto de contenido, al ya ser aplicado en otro estudio (PedreroPérez et al., 2018), como psicométrica, al lograr un ajuste satisfactorio en el análisis factorial confirmatorio de su estructura $(\mathrm{RMR}=0.012$; $\mathrm{GFI}=0.96$; $\mathrm{AGFI}=0.95$; $\mathrm{NFI}=$ 0.94; RFI=0.92; PGFI=0.73; PNFI= 0.79).

El instrumento está conformado por 20 ítems agrupados en 5 escalas (internet, móvil, videojuegos, mensajería instantánea y redes sociales). En cada escala se incluyen 4 preguntas relacionadas con conductas problemas: autopercepción de dedicación temporal excesiva; estimación de consumo excesivo en opinión de otros agentes; problemas para cesar la conducta; dificultad para interrumpir de forma voluntaria la conducta. Para este estudio, que forma parte de una investigación más amplía, el foco se situó en las 5 preguntas vinculadas a la valoración de los participantes respecto a la percepción de sus familias o allegados sobre el uso excesivo que los mismos realizaban en torno a las TIC -Tabla $1-$.

Tabla 1. Ítems del instrumento MULTICAGE-TIC para el análisis

\begin{tabular}{ll}
\hline Escala & Ítem \\
\hline Internet & ¿Se han quejado sus familiares de las horas que dedica a Internet? \\
\hline Móvil & $\begin{array}{l}\text { ¿Le han comentado en alguna ocasión, sus familiares o amigos, que utiliza } \\
\text { mucho el móvil para hablar o enviar mensajes? }\end{array}$ \\
\hline Videojuegos & $\begin{array}{l}\text { ¿Se queja su familia de que pasa demasiado tiempo jugando con la } \\
\text { videoconsola o el ordenador o el móvil? }\end{array}$ \\
\hline Mensajería & $\begin{array}{l}\text { ¿Le han dicho sus familiares o amigos que pasa demasiado tiempo } \\
\text { chateando a través del WhatsApp (u otro programa similar)? }\end{array}$ \\
\hline \multirow{2}{*}{ Redes sociales } & $\begin{array}{l}\text { ¿Le han dicho sus familiares o amigos que pasa demasiado tiempo } \\
\text { consultando y comunicándose a través del Facebook (o Twitter, o } \\
\text { Instagram o similares)? }\end{array}$ \\
\hline
\end{tabular}

Para responder a las cuestiones, se utilizó una escala tipo Likert con una valoración de 1 a 5 (1=Nunca; 2=Raramente; 3=Ocasionalmente; 4=Frecuentemente; 5=Siempre). Las puntuaciones que oscilan entre las valoraciones nunca y raramente se interpretaron como conducta no problemática; las que se sitúan en la valoración ocasionalmente como conducta de riesgo; la valoración de frecuentemente fue interpretada como conducta abusiva; y la valoración siempre como conducta dependiente. 


\subsection{Procedimiento}

Este estudio parte del interés sobre el uso problemático de las TIC y cómo la pandemia provocada por la COVID-19 ha podido influir respecto a dicho uso inadecuado teniendo en cuenta la percepción de las familias. La crisis sanitaria provocó un nuevo escenario en todas las esferas de la realidad, incluida la formación universitaria. Ante el confinamiento estipulado a nivel nacional, la Universidad de Málaga tuvo que adaptar su docencia al formato virtual durante el segundo cuatrimestre del curso 2019/2020. Esta situación influyó directamente en el aumento de consumo de los dispositivos tecnológicos para su proceso de enseñanza-aprendizaje, sumado al uso para interactuar a través de las TIC en una situación de aislamiento ocasionada por el encierro decretado. Para recoger la información, el alumnado que quiso participar respondió al instrumento MULTICAGE-TIC (a través de la aplicación LimeSurvey que permite que sea autoadministrado de forma telemática), de forma voluntaria y autónoma, al inicio del cuatrimestre (febrero 2020) y a mediación del mismo (abril 2020), durante la vigencia del estado de alarma en España y el correspondiente confinamiento.

\subsection{Análisis de datos}

Con el software SPSS v.25 se realizaron los análisis correspondientes para responder a los objetivos planteados. Se inició con un análisis exploratorio e inferencial de las respuestas a las preguntas seleccionadas en el pre-test y el post-test, considerando la muestra total y la existencia de diferencias significativas entre ambos momentos (prueba no paramétrica $\mathrm{W}$ de Wilcoxon). Tras ello, se realizaron análisis exploratorios e inferenciales en función de la variable sexo en el pre-test y el post-test, comprobando si existen diferencias significativas entre sexos en cada momento analizado (prueba no paramétrica U de Mann-Whitney).

\section{RESULTADOS}

Inicialmente, centramos la atención en el análisis de los resultados descriptivos obtenidos en el pre-test (antes del confinamiento) y el post-test (durante el confinamiento) tras la aplicación del instrumento. Incluimos en la tabla 2 los supuestos paramétricos de homocedasticidad y normalidad, así como el análisis inferencial respecto a la existencia de diferencias significativas entre ambos momentos.

Tabla 2. Estadísticos pre-test y post-test

\begin{tabular}{|c|c|c|c|c|c|c|c|c|}
\hline \multirow[t]{2}{*}{ TIC } & \multirow[t]{2}{*}{ Momento } & \multirow[t]{2}{*}{$\mathrm{M} \pm \mathrm{SD}$} & \multicolumn{2}{|c|}{$\begin{array}{c}\text { Kolmogorov- } \\
\text { Smirnov }\end{array}$} & \multicolumn{2}{|c|}{ Levene } & \multicolumn{2}{|c|}{ Wilcoxon } \\
\hline & & & $\mathrm{F}$ & p. & $\mathrm{F}$ & p. & $\mathrm{Z}$ & p. \\
\hline \multirow{2}{*}{ Internet } & Pre-1 & $2.69=$ & 0.183 & $0.000^{*}$ & \multirow{2}{*}{11.460} & \multirow{2}{*}{$0.001 *$} & \multirow{2}{*}{-8.426} & \multirow{2}{*}{$0.000^{*}$} \\
\hline & Post-test & $3.41 \pm 0.86$ & 0.247 & $0.000^{*}$ & & & & \\
\hline \multirow{2}{*}{ Móvil } & Pre-test & $2.98 \pm 1.20$ & 0.201 & $0.000^{*}$ & \multirow{2}{*}{11.229} & \multirow{2}{*}{$0.001^{*}$} & \multirow{2}{*}{-7.261} & \multirow{2}{*}{$0.000 *$} \\
\hline & Post-test & $3.79 \pm 0.78$ & 0.232 & $0.000^{*}$ & & & & \\
\hline \multirow{2}{*}{ Videojuegos } & Pre-test & $1.59 \pm 0.91$ & 0.358 & $0.000^{*}$ & \multirow{2}{*}{6.460} & \multirow{2}{*}{$0.012 *$} & \multirow{2}{*}{-10.619} & \multirow{2}{*}{$0.000 *$} \\
\hline & Post-test & $2.83 \pm 1.02$ & 0.324 & $0.000^{*}$ & & & & \\
\hline \multirow{2}{*}{$\begin{array}{l}\text { Mensajería } \\
\text { instantánea }\end{array}$} & Pre-test & $2.73 \pm 1.34$ & 0.175 & $0.000^{*}$ & \multirow{2}{*}{56.486} & \multirow{2}{*}{$0.000^{*}$} & \multirow{2}{*}{-8.241} & \multirow{2}{*}{$0.000 *$} \\
\hline & Post- & $4.19 \pm 0.78$ & 0.245 & $0.000^{*}$ & & & & \\
\hline \multirow{2}{*}{$\begin{array}{c}\text { Redes } \\
\text { sociales }\end{array}$} & & $2.74 \pm 1.29$ & 0.173 & $0.000^{*}$ & \multirow{2}{*}{29.609} & \multirow{2}{*}{$0.000^{*}$} & \multirow{2}{*}{-8.185} & \multirow{2}{*}{$0.000^{*}$} \\
\hline & Post-test & $3.20 \pm 0.90$ & 0.294 & $0.000^{*}$ & & & & \\
\hline
\end{tabular}

$*=\mathrm{p}<0.05$ 
Los datos reflejan un aumento del uso problemático en todas las escalas antes del confinamiento y durante el confinamiento provocado por el COVID-19, incrementando las medias entre 0.46 y 1.46 puntos. El móvil fue el dispositivo sobre el que más se quejaron las familias respecto a su uso antes del confinamiento (2.98 \pm 1.20$)$, mientras que la mensajería instantánea ocupo este lugar durante el confinamiento (4.19 \pm 0.78 ). Por el contra, la TIC que las familias consideran que los participantes usan en menor medida fueron los videojuegos (1.59 \pm 0.91 en el pre-test, $2.83 \pm 1.02$ en el post-test).

En cuanto a los supuestos paramétricos, los estadísticos de Leven y KolmogorovSmirnov (significatividad inferior a $0.05(\mathrm{p} \leq 0.05)$ en todas las escalas) no se ajustan a la normalidad, debiendo aplicar la prueba no paramétrica $\mathrm{W}$ de Wilcoxon para comparar las puntuaciones entre ambos momentos. Los resultados reflejan la existencia de diferencias significativas en todas las TIC respecto a la estimación de un uso excesivo en opinión de las familias, aumentando su consumo durante el confinamiento. De este modo, se pasa de un uso problemático en el pre-test (salvo los videojuegos donde no se reflejan que existan problemas) a un uso abusivo en el post-test en todas las escalas. Este crecimiento entre ambos momentos respecto al confinamiento puede verse reflejado en la figura 1 .

Figura 1. Comparativa pre-test / post-test

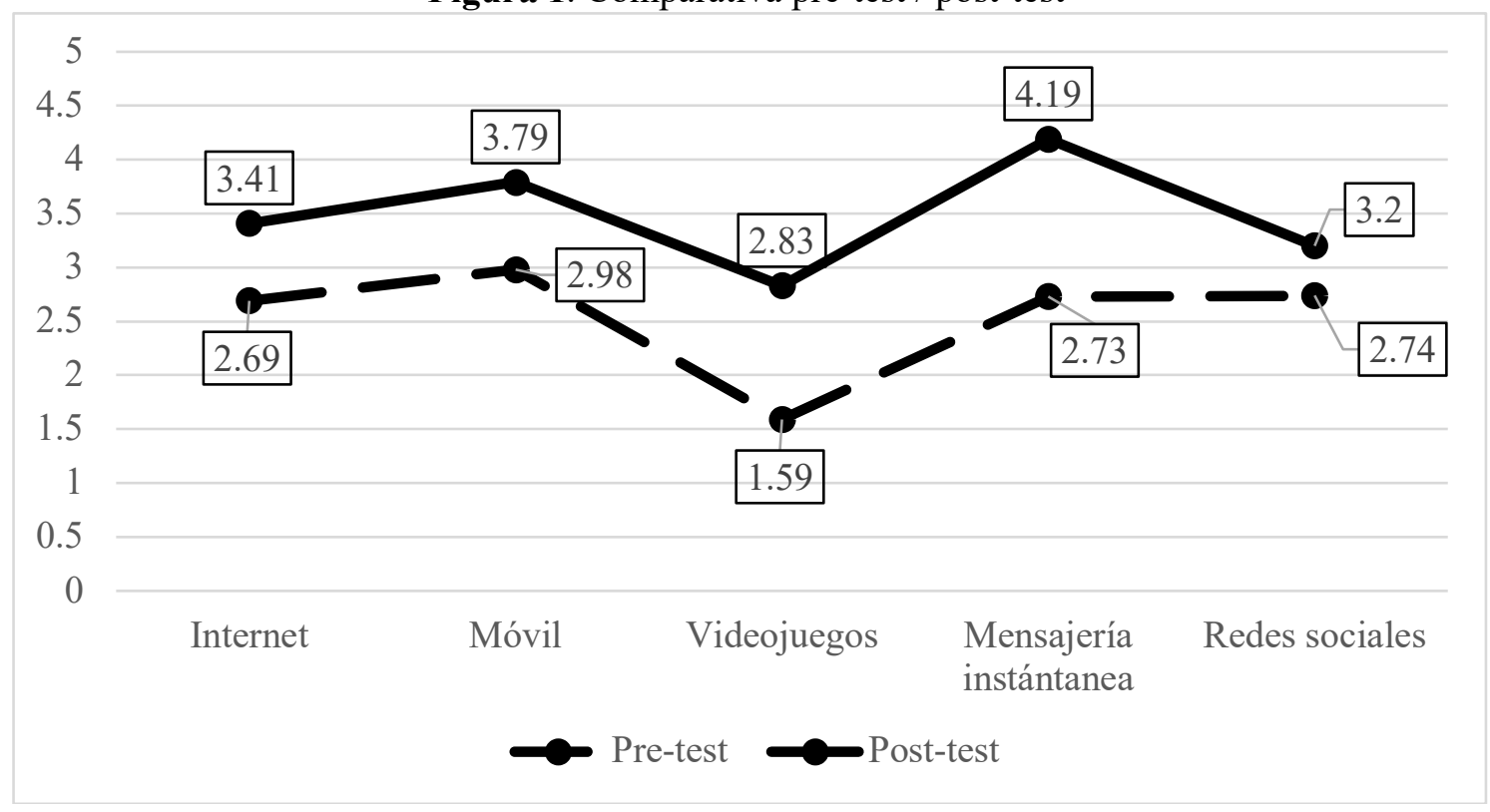

Seguidamente, vamos a profundizar en el análisis de la estimación del uso excesivo de tiempo en las TIC contempladas por parte de las familias. Comenzamos por la situación antes del confinamiento -Tabla 3-debido a la pandemia.

Los resultados indican que las familias de los participantes masculinos tenían una percepción mayor de un uso excesivo de las TIC en todas las escalas antes de la pandemia, salvo en el uso del móvil (3.02 \pm 1.31 de las mujeres por $2.88 \pm 0.82$ de los hombres). No obstante, ambos sexos se sitúan en valoraciones de uso problemático en todas las escalas a excepción de los videojuegos, donde tanto hombres como mujeres reflejan un uso no problemático ( $1.95 \pm 0.90$ hombres y $1.45 \pm 0.88$ mujeres). Atendiendo a la existencia de diferencias significativas, se aplicó la prueba no paramétrica U de Mann-Whitney al no ajustarse los datos a la normalidad (Leven, $\mathrm{p} \leq 0.05$ en 3 de las 5 escalas; KolmogorovSmirnov, $\mathrm{p} \leq 0.05$ en todas las escalas). Es relevante subrayar que solo se producen 
diferencias significativas antes de la pandemia en los videojuegos. Si bien es cierto que, en ambos casos, el tiempo de uso es considerado no problemático, se alcanza una diferencia de 0.50 puntos, estando los hombres más próximos a traspasar la consideración de uso no problemático al consumo de riesgo.

Tabla 3. Estadísticos pre-test en función de la variable sexo

\begin{tabular}{|c|c|c|c|c|c|c|c|}
\hline \multirow[t]{2}{*}{ TIC } & \multirow[t]{2}{*}{ Sexo } & \multirow[t]{2}{*}{$\mathrm{M} \pm \mathrm{SD}$} & \multicolumn{2}{|c|}{$\begin{array}{l}\text { Kolmogorov- } \\
\text { Smirnov }\end{array}$} & \multicolumn{2}{|c|}{ Levene } & \multirow{2}{*}{$\begin{array}{c}\text { Mann- } \\
\text { Whitney } \\
\text { p. }\end{array}$} \\
\hline & & & $\mathrm{F}$ & p. & $\mathrm{F}$ & p. & \\
\hline \multirow{2}{*}{ Internet } & Hombre & $2.78 \pm 0.77$ & 0.290 & $0.000 *$ & \multirow{2}{*}{16.560} & \multirow{2}{*}{$0.000^{*}$} & \multirow{2}{*}{0.432} \\
\hline & Mujer & $2.66 \pm 1.27$ & 0.157 & $0.000 *$ & & & \\
\hline \multirow{2}{*}{ Móvil } & Hombre & $2.88 \pm 0.82$ & 0.360 & $0.000 *$ & \multirow{2}{*}{17.981} & \multirow{2}{*}{$0.000^{*}$} & \multirow{2}{*}{0.361} \\
\hline & Mujer & $3.02 \pm 1.31$ & 0.175 & $0.000^{*}$ & & & \\
\hline \multirow{2}{*}{ Videojuegos } & Hombre & $1.95 \pm 0.90$ & 0.303 & $0.000^{*}$ & \multirow{2}{*}{0.106} & \multirow{2}{*}{0.746} & \multirow{2}{*}{$0.000^{*}$} \\
\hline & Mujer & $1.45 \pm 0.88$ & 0.423 & $0.000 *$ & & & \\
\hline \multirow{2}{*}{$\begin{array}{l}\text { Mensajería } \\
\text { instantánea }\end{array}$} & Hombre & $2.78 \pm 1.12$ & 0.280 & $0.000 *$ & \multirow{2}{*}{6.106} & \multirow{2}{*}{$0.015^{*}$} & \multirow{2}{*}{0.734} \\
\hline & Mujer & $2.72 \pm 1.41$ & 0.178 & $0.000 *$ & & & \\
\hline \multirow{2}{*}{$\begin{array}{c}\text { Redes } \\
\text { sociales }\end{array}$} & Hombre & $2.75 \pm 1.37$ & 0.208 & $0.000^{*}$ & \multirow{2}{*}{0.323} & \multirow{2}{*}{0.571} & \multirow{2}{*}{0.925} \\
\hline & Mujer & $2.74 \pm 1.27$ & 0.159 & $0.000 *$ & & & \\
\hline
\end{tabular}

Pasamos a analizar los efectos de la pandemia y el confinamiento en la percepción de las familias sobre el uso excesivo de las TIC -Tabla 4-.

Tabla 4. Estadísticos post-test en función de la variable sexo

\begin{tabular}{cccccccc}
\hline \multirow{2}{*}{ TIC } & \multirow{2}{*}{ Sexo } & \multirow{2}{*}{$\pm \mathrm{SD}$} & \multicolumn{2}{c}{ Kolmogorov- } & \multicolumn{2}{c}{ Levene } & \multirow{2}{*}{\begin{tabular}{c} 
Mann- \\
\cline { 3 - 7 }
\end{tabular}} \\
\cline { 3 - 8 } Shitney
\end{tabular}

Los datos reportan un cambio en las percepciones de las familias respecto al consumo de las TIC, siendo ahora las puntuaciones del sexo femenino mayores en 3 escalas (internet, móvil y mensajería instantánea) respecto a los hombres. Respecto a los usos problemático, el consumo no es de riesgo para las mujeres en los videojuegos (2.34 \pm 0.63$)$. Si pasa a ser problemático en internet para ambos sexos $(3.30 \pm 0.65$ hombres y $3.45 \pm 0.92$ mujeres), en el móvil y la mensajería instantánea para los hombres $(3.08 \pm 0.57$ y $3.30 \pm 0.69$, respectivamente), y en redes sociales para ambos $(3.25 \pm 0.98$ hombres y $3.18 \pm 0.88$ mujeres). Destacar, el consumo abusivo de las mujeres respecto al móvil (4.06 $\pm 0.67 \mathrm{y}$ de los hombres en los videojuegos (4.15 \pm 0.58$)$, y el uso dependiente de las mujeres en la mensajería instantánea $(4.52 \pm 0.50)$. 
En lo que respecta a la existencia de diferencias significativas, los datos no se ajustan a la normalidad (Leven, $\mathrm{p} \leq 0.05$ en 3 de las 5 escalas; Kolmogorov-Smirnov, $\mathrm{p} \leq$ 0.05 en todas las escalas), por lo que se vuelve a utilizar la prueba no paramétrica $U$ de Mann-Whitney. Durante el confinamiento, se aprecian diferencias significativas en el consumo del móvil, los videojuegos y la mensajería instantánea. En el móvil, los hombres tienen un uso problemático frente al consumo abusivo de las mujeres; en la mensajería instantánea, el sexo femenino lo consume de forma dependiente mientras que en el sexo masculino el uso es problemático; en los videojuegos, los hombres reflejan un consumo dependiente mientras que las mujeres se sitúan en un uso no problemático.

Para cerrar este apartado, vamos a reflejar las puntuaciones medias-Figura 2- de ambos sexos, antes y durante el confinamiento.

Figura 2. Comparativa pre-test / post-test en función de la variable sexo

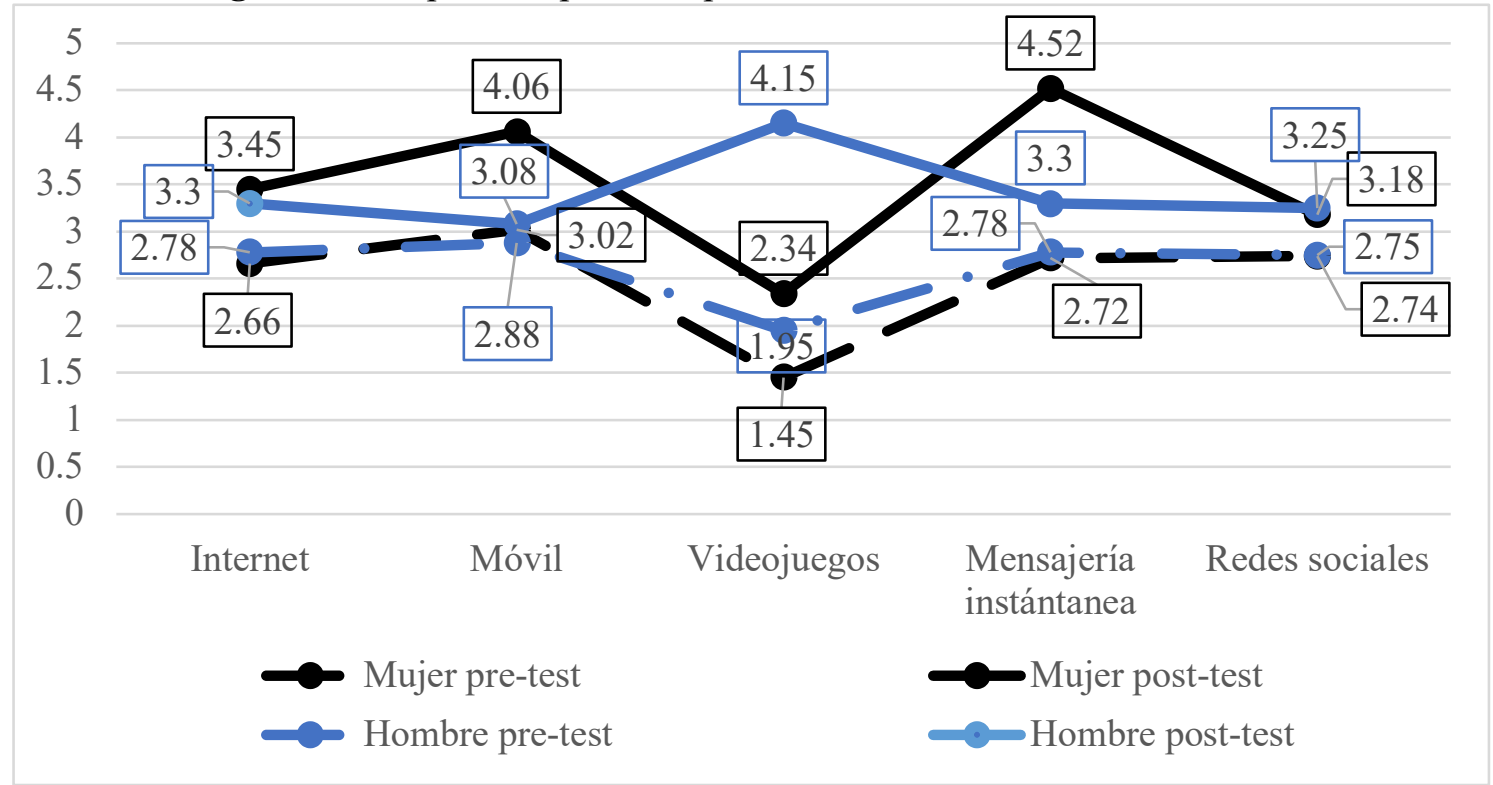

Como se observa en el gráfico, las puntuaciones de las mujeres han seguido una distribución semejante en ambos momentos, aumentando las puntuaciones en todos los casos. Por el contrario, las puntuaciones de los hombres no han seguido una distribución igualitaria. En su caso, salvo los videojuegos donde sí se aprecia una gran diferencia, las puntuaciones en el resto de TIC no difieren mucho entre sí entre los resultados de antes de la pandemia y después de la pandemia. Así, podemos reflejar que, en el caso de los hombres, las familias perciben un incremento excesivo del uso de los videojuegos, no aumentando excesivamente en el resto. En cuanto a las mujeres, las familias si denotan un aumento en todas las TIC a consecuencia del confinamiento derivado de la pandemia.

\section{DISCUSIÓN Y CONCLUSIONES}

Los hallazgos reflejan un incremento en la estimación de las familias del uso excesivo de las TIC a causa de la pandemia en la muestra objeto de estudio. Antes de la pandemia, los participantes reflejaban que sus familias percibían un uso problemático general de las TIC consideradas (salvo en los videojuegos), aumentando estas percepciones durante el confinamiento. Estos resultados coinciden con otros estudios 
(Dong et al., 2020; Lemenager et al., 2021; Wang et al., 2020) que también reseñaron un consumo mayor de las TIC asociado a la pandemia.

Atendiendo a los resultados por sexos, los datos reflejan que los hombres tenían un uso más problemático con las TIC que las mujeres antes del confinamiento provocado por el COVID-19, según la percepción de sus familias. Estos datos cambiaron a raíz del aislamiento provocado por el confinamiento, aumentando el uso excesivo en las mujeres respecto a los hombres en opinión de sus familias y allegados. Para profundizar en ello, comentamos los resultados de los diferentes elementos TIC considerados.

El uso de internet era problemático para ambos sexos según sus familias, aunque ligeramente superior para los hombres antes de la pandemia. Durante el confinamiento, se revierten la situación y son las mujeres quienes tienen un consumo mayor en opinión de sus familias. No obstante, no se hallan diferencias significativas en ninguno de los dos momentos, coincidiendo con el estudio de Fernández et al. (2020) y contrariando otras investigaciones (Peris et al., 2020).

El móvil, atendiendo a las consideraciones de familias y allegados, es más utilizado por las mujeres en ambos momentos, produciéndose diferencias significativas durante el confinamiento al alcanzar estas un uso abusivo de dicho dispositivo. Estos datos confirman lo hallado en otros estudios (Ballester et al., 2020; Chóliz et al., 2016; Long et al., 2016) donde las mujeres registran una mayor adicción respecto al teléfono móvil. Vinculado a este consumo, encontramos que el uso de la mensajería instantánea llega a ser considerado por las familias como dependiente para las mujeres en pleno confinamiento, mientras que para los hombres prevalece el uso problemático.

El uso de las redes sociales es semejante para ambos sexos en los dos momentos contemplados, ubicándose en un uso problemático en opinión de las familias. Esto contradice estudios que señalan un mayor consumo de las mujeres (Gómez et al., 2017; Peris et al., 2018).

En cuanto a los videojuegos, las familias de las mujeres no perciben riesgo en el consumo de los videojuegos, mientras que las de los hombres pasan de uso no problemático antes de la pandemia a uno abusivo durante el confinamiento, coincidiendo con el trabajo de Desai et al. (2010), que señala la mayor atracción de los hombres por los videojuegos.

Como podemos ver, la pandemia del COVID-19 ha influido en un mayor consumo de las TIC en opinión de las familias. Este hecho conlleva una necesaria reflexión a nivel de la praxis docente. Estamos ante un aumento de conductas adictivas respecto a las tecnologías y en un escenario en el que las familias no tienen, en muchos casos, la competencia digital suficiente para acompañar adecuadamente en un uso responsable de las TIC. En este sentido, tan importante es mejorar la competencia digital de los docentes en formación y en activo, como mejorar los canales de comunicación y las vías de relación entre el profesorado y las familias. Las tecnologías tienen muchas posibilidades y depende del uso que se les den, por lo que es necesario trabajar y formarnos para responder satisfactoriamente a nuestra labor educativa, como para ayudar a las familias a gestionar los usos problemáticos de las TIC en los discentes.

En cuanto a las limitaciones, señalamos la conveniencia de cuestionar a las propias familias aspectos concretos sobre el consumo de TIC y cuáles son los criterios para estipular un uso inadecuado a nivel de tiempo o dependencia. Como futuras líneas de investigación, es preciso acometer un estudio de la situación cuando se solvente la crisis 
sanitaria, para analizar el impacto en el uso y consumo de TIC tras lo vivido a causa de la pandemia.

\section{REFERENCIAS BIBLIOGRÁFICAS}

Ballester, M.L., Simó, C., Chover, E., Saus, C., Casal, C., \& Martínez, A. (2020). SelfPerception of Dependence as an Indicator of Smartphone AddictionEstablishment of a Cutoff Point in the SPAI-Spain Inventory. International Journal of Environmental Research and Public Health, 17(11), e3838. https://doi.org/10.3390/ijerph17113838

Bringué, X., Sádaba, C. y Sanjurjo, E. (2013). Menores y ocio digital en el siglo XXI. Análisis exploratorio de perfiles de usuarios de videojuegos en España. Bordón. Revista De Pedagogía, 65(1), 147-164.

Cánovas, P. y Sahuquillo, P. (2014). La familia como contexto de desarrollo y educación. En P. Cánovas y P. Sahuquillo ( Coords.), Familias y menores. Retos y propuestas pedagógicas (pp. 24-38). Tirant Humanidades.

Chóliz, M., Pinto, L., Phansalkar, S., Corr, E., Mujjahid, A., Flores, C. y Barrientos, P. (2016). Development of a Brief Multicultural Version of the Test of Mobile Phone Dependence (TMDbrief) Questionnaire. Frontiers in Psychology, 7, 650. https://doi.org/10.3389/fpsyg.2016.00650

Desai, R.A., Krishnan-Sarin, S., Cavallo, D. y Potenza, M.N. (2010). Video-Gaming Among High School Students: Health Correlates, Gender Differences, and Problematic Gaming. Pediatrics, 126(6), e1414-e1424. https://doi.org/10.1542/peds.2009-2706

Dong, H., Yang, F., Lu, X. y Hao, W. (2020). Internet Addiction and Related Psychological Factors Among Children and Adolescents in China During the Coronavirus Disease 2019 (COVID-19) Epidemic. Frontiers in Psychiatry, 11, 751. https://doi.org/10.3389/fpsyt.2020.00751

Fernández, S., Belzunegui, Á., Pastor, I. y Valls, F. (2020). Compulsive Internet and Prevalence Substance Use among Spanish Adolescents. International Journal of Environmental Research and Public Health, 17(23), e8747. https://doi.org/10.3390/ijerph17238747

García-Peñalvo, F.J. y Corell, A. (2020). La CoVId-19: ¿enzima de la transformación digital de la docencia o reflejo de una crisis metodológica y competencial en la educación superior?. Campus Virtuales, 9(2), 83-98.

Gómez, P., Rial, A., Braña, T., Golpe, S. y Varela, J. (2017). Screening of problematic internet use among Spanish adolescents: prevalence and related variables. Cyberpsychology, Behavior, and Social Networking, 20(4), 259-267. https://doi.org/10.1089/cyber.2016.0262

González-Fernández, N., Ramírez, A. y Aguaded, I. (2019). Alfabetización mediática en escenarios familiares. Diagnóstico, necesidades y propuesta. Education in the Knowledge Society, 20, 1-13. https://doi.org/10.14201/eks2019_20_a11

Gonzálvez, M.T., Espada, J.P. y Tejeiro, R. (2017). El uso problemático de videojuegos está relacionado con problemas emocionales en adolescentes. Adicciones, 29(3), 180-185. https://doi.org/10.20882/adicciones.745

Hodges, C., Moore, S., Lockee, B., Trust, T. y Bond, A. (2020). The difference between emergency remote teaching and online learning. Educause Review. https://bit.ly/3vNq4m3 
Instituto Nacional de Estadística (2020). Encuesta de población activa. Segundo trimestre.

https://www.mites.gob.es/ITSS/ITSS/ITSS Descargas/Atencion_ciudadano/Nor mativa documentacion/Otra docum/epa0220.pdf

Labrador, F., Requesens, A. y Helguera, M. (2018). Guía para padres y educadores sobre el uso seguro de Internet, móviles y videojuegos. Fundación Gaudium, Obra Social Caja Madrid y Defensor del Menor de la Comunidad de Madrid.

Lemenager, T., Neissner, M., Koopmann, A., Reinhard, I., Georgiadou, E., Müller, A., Kiefer, F. y Hillemacher, T. (2021). Covid-19 lockdown restrictions and online media consumption in Germany. International Journal of Environmental Research and Public Health, 18(1), e14. https://doi.org/10.3390/ijerph18010014

Leoz, D. (2015). La afluencia de los medios de comunicación en el proceso de socialización y la importancia de la coeducación para la igualdad. Hachetetepé. Revista científica de Educación y Comunicación, (11), 131-140. https://doi.org/10.25267/Hachetetepe.2015.v2.i11.12

Long, J., Liu, T.Q., Liao, Y.H., Qi, C., He, H.Y., Chen, S.B. y Billieux, J. (2016). Prevalence and correlates of problematic smartphone use in a large random sample of Chinese undergraduates. BMC Psychiatry, 16, e408. https://doi.org/10.1186/s12888-016-1083-3

López, S. y González, C. (2017). Utilización didáctica dos videoxogos na escola e na familia: posibilidades e recomendacións. Revista galega de educación, (68), 1720.

Muros, B., Aragón, Y. y Bustos, A. (2013) La ocupación del tiempo libre de jóvenes en el uso de videojuegos y redes. Comunicar, (40), 31-39. https://doi.org/10.3916/C40-2013-02-03

Pedrero-Pérez, E.J., Ruiz-Sánchez, J.M., Rojo-Mota, G., Llanero-Luque, M., PedreroAguilar, J., Morales-Alonso, S. y Puerta-García, C. (2018). Tecnologías de la Información y la Comunicación (TIC): uso problemático de Internet, videojuegos, teléfonos móviles, mensajería instantánea y redes sociales mediante el MULTICAGE-TIC. $\quad$ Adicciones, $\quad 30(1), \quad$ 19-32. https://doi.org/10.20882/adicciones.806

Peris, M., Barrera, U., Schoeps, K., \& Montoya, I. (2020). Psychological Risk Factors that Predict Social Networking and Internet Addiction in Adolescents. International Journal of Environmental Research and Public Health, 17(12), e4598. https://doi.org/10.3390/ijerph17124598

Peris, M., Maganto, C. y Garaigordobil, M. (2018). Scale of risk of addiction to social networks and Internet for adolescents: reliability and validity (ERA-RSI). Rev. Psicol. Clin. Ninos Adolesc., 30-36. https://doi.org/10.21134/rpcna.2018.05.2.4.

Ricoy, M.C. y Ameneiros, A. (2016). Preferencias, dedicación y problemáticas generadas por los videojuegos: Una perspectiva de género. Revista Complutense de Educación, 27(3), 1291-1308.

Wang, Y.-Y., Long, J., Liu, Y.-H., Liu, T.-Q., \& Billieux, J. (2020). Factor structure and measurement invariance of the problematic mobile phone use questionnaire-short version across gender in Chinese adolescents and young adults. BMC Psychiatry, 20(1), 34. https://doi.org/10.1186/s12888-020-2449-0 